\title{
1997-2000 CCD astrometric observations of Saturn's satellites and comparison with theories ${ }^{\star}$
}

\author{
R. C. Qiao ${ }^{1,2}$, K. X. Shen ${ }^{1,2}$, D. Harper ${ }^{3}$, and J. R. Liu ${ }^{1,2}$ \\ 1 National Time Service Center, The Chinese Academy of Science, PO Box 18, Lintong, Shaanxi 710600, PR China \\ 2 United Laboratory for Optical Astrometry, The Chinese Academy of Science, PR China \\ 3 Wellcome Trust Sanger Institute, Wellcome Trust Genome Campus, Hinxton, Cambridge CB10 1SA, UK
}

Received 18 November 2003 / Accepted 16 April 2004

\begin{abstract}
As a continuation of the observing campaign 1994-1996 (Qiao et al. 1999), this paper presents 1167 new measurements of the positions of the major Saturnian satellites obtained using a CCD camera on the $1.56 \mathrm{~m}$ reflector at the Sheshan station near Shanghai during the period 1997-2000. The observations include the major satellites exclusive of the faintest satellite, Hyperion. The analytical theory TASS was chosen for astrometric calibration of the CCD and theory computation. Analysis of the data as inter-satellite positions shows that the observations of Tethys, Dione, Rhea, and Iapetus have a standard deviation about the mean of 0'06, which matches the accuracy of our earlier observations. However, for a few of the inner satellites, including Mimas, Enceladus, we have obtained poorer residuals up to 0.1 to 0.2 which we ascribe to lower quantum efficiency of the CCD.
\end{abstract}

Key words. planets and satellites: individual: saturn - astrometry

\section{Introduction}

In a previous paper (Qiao et al. 1999, hereafter referred to as Paper I), we reported an astrometric observing program of the major satellites of Saturn using a CCD camera on the $1.56 \mathrm{~m}$ reflector at the Sheshan station (E 121.18417, N 31.09611) of the Shanghai Astronomical Observatory. As a continuation of our observing campaigns of 1994-1996, we present 918 new observations obtained during the 1997-2000 oppositions.

All the observations were carried out with the similar CCD detector and same telescope under very similar conditions, so the procedures of observing, measuring and calibration calculation remain essentially the same as described in Paper I, and the reader is suggested to refer to the papers of Qian et al. (2003) and Tao et al. (2000) for details about the $\mathrm{CCD}$ detector and reflector.

The current paper is organized as following sections: in Sect. 2 the observations and reduction procedures are presented; in Sect. 3 the theory used for calculation of positions are chosen; in Sect. 4 the results and analysis are given. The conclusion is presented in the final section.

Send offprint requests to: R. C. Qiao,

e-mail: rcqiao@ntsc.ac.cn

* The data are only available in electronic form at the CDS via anonymous ftp to cdsarc.u-strasbg.fr $(130.79 .128 .5)$ or via http://cdsweb.u-strasbg.fr/cgi-bin/qcat?]/A+A/422/377

\section{Observation and reduction}

We were allocated 4-6 days of telescope time in each of the years 1997-2000. During this period, 11 nights of observations enabled us to obtain $249 \mathrm{CCD}$ images.

The CCD chip is an array of $1024 \times 1024$ pixels which has a field of view of only $4^{\prime} \times 4^{\prime}$. No reference stars can be observed in such a small field of view, so we must use the "bright moon method" (Shen et al. 2001; Harper et al. 1997) to determination the astrometric calibration parameters of the CCD.

The data (position angle $S_{\mathrm{c}} P$ and separation $S$ ) we give in this paper are referred to J2000 system. The time argument is the UTC.

\subsection{Calculation of astrometric corrections}

Following the example of Shen et al. (2002), some significant local effects (atmospheric refraction, stellar aberration and topocentric parallax) are taken into account in this paper. However, in Shen et al. (2002), the effects due to central projection and light-travel time between satellites were omitted because the maximum separation between the Uranian satellites was less than $55^{\prime \prime}$.

The reduction of astrometric CCD observations of planetary satellites has been described in detail by Vienne et al. (2001a,b) and Peng et al. (2002). Vienne et al. made a special discussion of central projection, which was neglected by 
Table 1. Calibration parameters for each night of observations.

\begin{tabular}{cccr}
\hline \hline Subset & Date & $\rho($ arcsec/pixel $)$ & $\delta P($ degree $)$ \\
\hline $1997 \mathrm{a}$ & 1997.09 .01 & 0.250929 & 14.8677 \\
$1997 \mathrm{~b}$ & 1997.09 .05 & 0.251146 & 14.7476 \\
$1998 \mathrm{a}$ & 1998.11 .07 & 0.251701 & 7.8102 \\
$1998 \mathrm{~b}$ & 1998.11 .08 & 0.251652 & 7.9718 \\
$1998 \mathrm{c}$ & 1998.11 .09 & 0.251408 & -14.9194 \\
$1998 \mathrm{~d}$ & 1998.11 .10 & 0.251705 & -3.1411 \\
$1999 \mathrm{a}$ & 1999.11 .12 & 0.251629 & 5.9487 \\
$1999 \mathrm{~b}$ & 1999.11 .13 & 0.251673 & 5.9462 \\
$2000 \mathrm{a}$ & 2000.11 .17 & 0.251629 & -7.3297 \\
$2000 \mathrm{~b}$ & 2000.11 .20 & 0.251705 & -7.3364 \\
$2000 \mathrm{c}$ & 2000.11 .21 & 0.251658 & -7.3687 \\
\hline
\end{tabular}

most authors in the past. As Vienne pointed out, although Shen et al. (2001) applied the correction for central projection, they made the incorrect assumption that the reference satellite can be treated as if it is the centre of the projection. In the present work, we have rigorously applied the iterative correction procedure described by Vienne et al. (2001b).

The results of reducing the observations show that the mean precision level for inter-satellite astrometry is now about $0 .^{\prime} 08$. As Vienne et al. have shown, the effect of central projection depends strongly upon the declination of the observed bodies (approximated by $s^{2} \tan \delta_{\mathrm{c}}$ ). We have found that the effects of central projection do not exceed 0. '005 for most subsets of observations which we report in this paper. In the subsets for the year 2000 when the declination of Saturn reaches $17^{\circ}$, the correction for central projection is up to $0 . ' 007$.

\subsection{Calibration and theory}

As noted by Qiao et al. (1999), the CCD chip was remounted on the reflector at the Sheshan station each night. Consequently, it was necessary to run a separate calibration determination for each night.

In the paper of Shen et al. (2001), a detailed description and analysis of the "brighter moon calibration" method was given; this method was also quite applied in the present reduction.

We adopt the form of polar coordinates (position angle $P$ and separation $S$ ) for the data in our measurements. The corresponding calibration parameters are denoted by $\delta P$ and $\rho$. Table 1 gives the calibration parameters for each subset.

The observations in this paper are also published in the form of raw pixel coordinates in order that future investigators can make direct use of the CCD images if they wish.

Several analytical theories exist for the major satellites of Saturn. We prefer to use TASS1.7 (Vienne \& Duriez 1995; Duriez \& Vienne 1997), because this theory has been verified by Shen et al. (2001) as the best current dynamical theory. The positions of Saturn were calculated from the JPL DE200 planetary ephemeris.
Table 2. Statistics of O-C residuals (in mas) from TASS for each of the Saturnian satellite on each of the observation subset, these residuals are relative to Titan or Rhea. $N$ gives the number of observations used for each satellites. $\mu$ is the mean residuals. $\sigma$ is the standard deviation (rms) about mean.

\begin{tabular}{|c|c|c|c|c|c|}
\hline \multirow[b]{2}{*}{ 1997a(Sat. - Titan) } & \multirow[b]{2}{*}{$N$} & \multicolumn{2}{|c|}{ Position angle } & \multicolumn{2}{|l|}{ "Separation } \\
\hline & & $\mu$ & $\sigma$ & $\mu$ & $\sigma$ \\
\hline Enceladus & 19 & $-40.6 \pm 24.7$ & 99.6 & $-103.8 \pm 28.8$ & 71.2 \\
\hline Tethys & 20 & $15.2 \pm 14.8$ & 64.4 & $-1.8 \pm 12.2$ & 54.6 \\
\hline Dione & 20 & $9.0 \pm 15.7$ & 69.6 & $28.4 \pm 16.3$ & 67.3 \\
\hline Rhea & 20 & $-17.2 \pm 11.4$ & 48.0 & $-29.1 \pm 12.8$ & 49.2 \\
\hline \multicolumn{6}{|l|}{ 1997b(Sat. - Rhea) } \\
\hline Tethys & 8 & $28.3 \pm 16.4$ & 36.8 & $-20.9 \pm 56.0$ & 156.9 \\
\hline Dione & 8 & $-52.6 \pm 55.8$ & 148.7 & $80.2 \pm 98.0$ & 265.2 \\
\hline \multicolumn{6}{|l|}{ 1998a(Sat. - Titan) } \\
\hline Mimas & 6 & $-240.3 \pm 106.6$ & 102.1 & $34.3 \pm 34.7$ & 77.7 \\
\hline Enceladus & 14 & $-4.5 \pm 7.3$ & 26.9 & $-36.9 \pm 23.3$ & 79.2 \\
\hline Tethys & 14 & $-8.1 \pm 9.8$ & 35.9 & $0.2 \pm 15.6$ & 58.6 \\
\hline Dione & 14 & $-18.3 \pm 12.1$ & 41.3 & $54.1 \pm 18.7$ & 44.6 \\
\hline Rhea & 13 & $-2.6 \pm 12.4$ & 44.5 & $60.8 \pm 20.6$ & 42.3 \\
\hline \multicolumn{6}{|l|}{ 1998b(Sat. - Titan) } \\
\hline Mimas & 14 & $-183.3 \pm 60.2$ & 131.1 & $45.0 \pm 17.3$ & 46.5 \\
\hline Enceladus & 16 & $35.3 \pm 18.0$ & 63.6 & $80.3 \pm 31.9$ & 99.0 \\
\hline Tethys & 22 & $-13.1 \pm 10.2$ & 46.2 & $15.6 \pm 11.0$ & 49.3 \\
\hline Dione & 22 & $12.5 \pm 11.3$ & 50.4 & $47.7 \pm 15.7$ & 56.2 \\
\hline Rhea & 22 & $4.8 \pm 9.6$ & 44.8 & $66.7 \pm 16.8$ & 41.8 \\
\hline \multicolumn{6}{|l|}{ 1998c(Sat. - Titan) } \\
\hline Enceladus & 14 & $224.2 \pm 71.5$ & 146.0 & $-248.7 \pm 74.4$ & 124.8 \\
\hline Tethys & 14 & $-171.3 \pm 77.9$ & 235.6 & $-19.8 \pm 47.2$ & 175.5 \\
\hline Dione & 14 & $52.2 \pm 24.7$ & 76.1 & $26.7 \pm 29.5$ & 107.0 \\
\hline Rhea & 14 & $93.8 \pm 39.2$ & 112.9 & $110.9 \pm 41.3$ & 107.6 \\
\hline \multicolumn{6}{|l|}{ 1998d(Sat. - Titan) } \\
\hline Enceladus & 10 & $-104.5 \pm 43.8$ & 90.8 & $-171.4 \pm 57.7$ & 60.7 \\
\hline Tethys & 13 & $11.8 \pm 13.4$ & 46.7 & $8.3 \pm 15.7$ & 55.9 \\
\hline Dione & 13 & $-8.4 \pm 15.7$ & 55.9 & $62.1 \pm 26.6$ & 73.2 \\
\hline Rhea & 13 & $-20.0 \pm 11.8$ & 37.5 & $53.3 \pm 21.4$ & 55.6 \\
\hline \multicolumn{6}{|l|}{ 1999a(Sat. - Rhea) } \\
\hline Mimas & 7 & $61.6 \pm 43.8$ & 90.8 & $-325.6 \pm 131.3$ & 121.1 \\
\hline Encoladus & 20 & $-103.0 \pm 34.0$ & 111.5 & $-90.6 \pm 40.2$ & 155.4 \\
\hline Tethys & 21 & $-7.0 \pm 10.2$ & 46.2 & $3.4 \pm 14.8$ & 68.0 \\
\hline Dione & 21 & $5.3 \pm 19.0$ & 86.9 & $38.6 \pm 26.9$ & 116.9 \\
\hline \multicolumn{6}{|l|}{ 1999b(Sat. - Rhea) } \\
\hline Mimas & 13 & $-181.5 \pm 55.9$ & 87.7 & $23.1 \pm 21.3$ & 73.1 \\
\hline Encoladus & 12 & $-140.6 \pm 46.1$ & 75.8 & $-363.4 \pm 112.9$ & 144.7 \\
\hline Tethys & 13 & $14.2 \pm 11.2$ & 37.7 & $5.3 \pm 7.6$ & 26.7 \\
\hline Dione & 13 & $-8.2 \pm 7.5$ & 25.6 & $41.6 \pm 14.5$ & 31.4 \\
\hline \multicolumn{6}{|l|}{ 2000a(Sat. - Titan) } \\
\hline Tethys & 28 & $-55.6 \pm 14.0$ & 49.4 & $-109.1 \pm 33.3$ & 138.5 \\
\hline Dione & 27 & $125.5 \pm 26.5$ & 56.2 & $45.5 \pm 23.0$ & 110.5 \\
\hline Rhea & 29 & $-82.6 \pm 17.1$ & 41.1 & $121.5 \pm 25.2$ & 60.7 \\
\hline Iapetus & 20 & $-116.1 \pm 28.3$ & 50.8 & $120.7 \pm 28.7$ & 43.8 \\
\hline \multicolumn{6}{|l|}{ 2000b(Sat. - Titan) } \\
\hline Enceladus & 18 & $252.1 \pm 79.7$ & 225.5 & $-278.1 \pm 76.1$ & 164.1 \\
\hline Tethys & 34 & $72.8 \pm 30.8$ & 164.5 & $61.4 \pm 19.2$ & 93.5 \\
\hline Dione & 46 & $-63.9 \pm 12.8$ & 59.1 & $-59.8 \pm 14.4$ & 77.2 \\
\hline Rhea & 46 & $-69.3 \pm 12.8$ & 52.2 & $61.4 \pm 15.1$ & 81.9 \\
\hline Iapetus & 46 & $-60.1 \pm 11.4$ & 48.5 & $30.5 \pm 7.2$ & 37.9 \\
\hline \multicolumn{6}{|l|}{$2000 c($ Sat. - Titan) } \\
\hline Mimas & 4 & $-230.4 \pm 131.4$ & 126.3 & $216.1 \pm 118.1$ & 95.4 \\
\hline Enceladus & 18 & $-257.0 \pm 72.7$ & 170.5 & $-290.1 \pm 79.6$ & 172.7 \\
\hline Tethys & 49 & $-91.4 \pm 18.9$ & 95.9 & $-26.7 \pm 12.1$ & 80.7 \\
\hline Dione & 49 & $44.5 \pm 12.1$ & 70.8 & $81.0 \pm 16.9$ & 84.1 \\
\hline Rhea & 28 & $67.2 \pm 16.9$ & 58.9 & $200.1 \pm 39.8$ & 66.2 \\
\hline
\end{tabular}




\section{Results and analysis}

We have calculated the mean and root-mean-square of observed-minus-calculated $(\mathrm{O}-\mathrm{C})$ residuals of the position angle and separation $\left(S_{\mathrm{c}} \Delta P, \Delta S\right)$ of each satellite relative to Titan or Rhea. The rejection level was $0 . ' 8$. The results are shown in Table 2.

In Table 2 we can observe that the larger residuals appear for a few of the inner satellites, especially, for those in the frames taken in 2000.

In 1998, the CCD chip used at the Sheshan station was damaged due to accident. It was replaced by another CCD chip which had a lower quantum efficiency, resulting in reduced quality of the images of some satellites obtained.

The calculations show that the root-mean-residual of O-C residual for Tethys, Dione, Rhea and Iapetus is about $0{ }^{\prime} 06$. For these satellites, the observations match the accuracy of the data published in Paper I. However, for the inner satellites close to the primary, especially Mimas and Enceladus, the residuals are as large as 0.10 .

The situation was improved in 2002 when a new $2048 \times$ 2048 pixel CCD chip was installed at the Sheshan station.

\section{Conclusion}

The observing run during 1997-2000 is a continuation of our observing program, which was initiated in 1994, aimed at obtaining astrometric data on the major satellites of Saturn using $\mathrm{CCD}$ technique on the 1.56-m reflector at the Sheshan station near Shanghai observatory.

In the implementation of our program since 1994, we have been in close collaboration with colleagues at Queen Mary and Westfield College (QMW) (Harper et al. 1997; Shen et al. 2002) and the Royal Greenwich Observatory(RGO) (Taylor \& Shen 1988), who conducted a parallel observing campaign which provided a significant number of highquality CCD observations of the Saturnian satellites. We were saddened to learn recently that the 1-m Jacobus Kapteyn telescope on La Palma is no longer also available for this type of observation.

We shall continue our astrometric campaign in the years 2003-2005, making full use of the new larger CCD chip installed on the 1.56-m reflector at the Sheshan station. Our colleague Dr. Peng, of Jinan University in Guangzhou, will also make astrometric observations of the satellites of Saturn and Uranus using the 1-m reflector at Yunnaan Observatory.

Acknowledgements. The authors wish to thank again the staff at the Sheshan station of the Shanghai Observatory for their assistance and especially to Dr. J. Tao, Profs. B. C. Qian and H. J. Pan for providing us many conveniences throughout our observing run. We are very grateful to Dr. Vienne and Dr. Peng for their valuable discussion about the astrometric correction. We should also mention the kind assistance which we received from Dr. E. M. Standish in our use of the JPL DE200 ephemeris.

This work was carried with financial support from the National Science Foundation of China (NSFC).

\section{References}

Duriez, L., \& Vienne, A. 1991, A\&A, 243, 263

Harper, D., Murray, C. D., Beurle, K., et al. 1997, A\&A, 121, 65

Peng, Q. Y., Vienne, A., \& Shen, K. X. 2002, A\&A, 383, 296

Qian, B. C., \& Tao, J. 2003, PASP, 115, 490

Qiao, R. C., Shen, K. X., Liu, J. R., \& Harper, D. 1999, A\&AS, 137, 1

Shen, K. X., Dourneau, G., Qiao, R. C., \& Liu, J. R. 2001, A\&A, 367, 1061

Shen, K. X., Qiao, R. C., Harper, D., Hadjifotinou, K. G., \& Liu, J. R. 2002, A\&A, 391, 775

Tao, J., Qian, B. C., Gu, M. F., \& Song, B. J. 2000, Plan. Space Sci., 48,153

Taylor, D. B., \& Shen, K. X. 1998, A\&A, 200, 269

Vienne, A., \& Duriez, L. 1995, A\&A, 297, 588

Vienne, A., Thuillot, W., Veiga, C. H., Arlot, J. E., \& Vieira, R. 2001a, A\&A, 380, 727

Vienne, A., Thuillot, W., \& Arlot, J. E. 2001b, Note Scientifique et Technique, de 1'I.M.C.C.E., S077 